\title{
IMPACT OF CUTTING FORCES AND CHIP MICROSTRUCTURE IN HIGH SPEED MACHINING OF CARBON FIBER - EPOXY COMPOSITE TUBE
}

\begin{abstract}
Carbon fiber reinforced polymeric (CFRP) composite materials are widely used in aerospace, automobile and biomedical industries due to their high strength to weight ratio, corrosion resistance and durability. High speed machining (HSM) of CFRP material is needed to study the impact of cutting parameters on cutting forces and chip microstructure which offer vital inputs to the machinability and deformation characteristics of the material. In this work, the orthogonal machining of CFRP was conducted by varying the cutting parameters such as cutting speed and feed rate at high cutting speed/feed rate ranges up to $346 \mathrm{~m} / \mathrm{min} / 0.446 \mathrm{~mm} / \mathrm{rev}$. The impact of the cutting parameters on cutting forces (principal cutting, feed and thrust forces) and chip microstructure were analyzed. A significant impact on thrust forces and chip segmentation pattern was seen at higher feed rates and low cutting speeds.

Keywords: Composite materials, orthogonal machining, cutting forces, chip microstructure
\end{abstract}

\section{Introduction}

Composite materials are increasingly being used by the aerospace, automobile and biomedical industries mainly due to their superior material properties such as high strength to weight ratio, corrosion resistance and durability. However, these materials are classified as difficult to machine materials, forcing industries to opt for near net shape manufacturing methods to create composite products. In reality, machining is an undesirable primary processing stage in a manufacturing process, especially in the production of complex product designs where dimensional accuracy and surface finish are critical factors. In this eventuality, Industries prefer HSM to achieve superior surface finish, higher production rates and lower manufacturing costs. Researchers have tended to work on composite machining in lower and trusted cutting ranges given the high costs in fabricating the CFRP materials. However HSM is needed to conduct a meaningful research work relevant to society in general and industry in particular.

In the past, machinability studies have dominated the experimental work on composite materials especially CFRP materials with primary focus on surface roughness, tool wear, tool life and cutting forces [1-3]. Teti [2002] presented a detailed account of various machinability issues with composite materials specifically mentioning that Poly crystalline diamond (PCD) tools were most suited for machining CFRP and Glass fiber reinforced polymers (GFRP) materials and ceramic based tools with their low thermal conductivity were the least preferred. Wang and Zhang [2003] asserted that fiber orientations affected the surface integrity during orthogonal machining and that the rake angle only marginally affected the surface roughness properties. Chung-Shin Chang [2006] compared the K and P type chamfered carbide tools and suggested a preference for the $\mathrm{K}$ type due to its superior hardness and wear resistance. Rafal Rusinek [2010] developed a new machining force model for the milling process of the epoxide-polymer matrix composite reinforced carbon fiber. Dandekar and Shin [2012] presented a detailed review of the modelling and machining of composites, concluding that numerical models are needed in selection of machining parameters like tool geometry and cutting conditions. Claus Dold et al [2012] compared the performance of ground and laser machined PCD tools in cutting CFRP material at a cutting speed of 200 $\mathrm{m} / \mathrm{min}$. Pecat et al [2012] investigated the surface integrity on a circumferential milling process of a unidirectional CFRP material observing a tendency for the material to show fiber bending towards the cutting direction. Rajasekaran et al [2012] optimized the machining parameters that provide better surface roughness using Taguchi methodology. Vijayan Krishnaraj et al [2012] studied the optimum cutting conditions in drilling of CFRP laminates using Taguchi method varying the spindle speed and feed rate, concluding that lower feed rates reduced the thrust force and delamination factors. Takeshi Yashiro et al [2013] in a work pertaining to temperature measurement during machining of CFRP concluded that its low thermal conductivity ensured its

\footnotetext{
* MOUNT ZION COLLEGE OF ENGINEERING AND TECHNOLOGY, PUDUKOTTAI, TAMILNADU, INDIA

** KCG COLLEGE OF TECHNOLOGY, CHENNAI, TAMILNADU, INDIA

*** SSN COLLEGE OF ENGINEERING, CHENNAI, INDIA

\# Corresponding author: y.allwinroy@gmail.com, allwin12001@mech.ssn.edu.in
} 
applicability in high speed milling even at high cutting speeds. Rentsch et al [2011] simulated the milling of CFRP material and evaluated the forces for $0^{\circ}$ and $90^{\circ}$ fiber orientations suggesting that only outer fibers were damaged during milling. Karpat and Polat [2013] developed a new mechanistic force model for double helix tools which can be used to improve double helix tool designs and optimize the milling process parameters.

The aim of the present work is to understand the impact of cutting parameters during HSM of CFRP material. In particular, effect of thrust and feed forces have been studied, of which little literature is available. Chip microstructure, which offers vital information on the material deformation behaviour, has been analyzed and the fiber and matrix fracture modes and patterns have been assessed at various cutting conditions. The results give insights into the uniqueness of the CFRP material and their deformation characteristics in a machining process.

\section{Experimental Work}

The carbon fiber tube was fabricated through a filament winding process with epoxy resin as the matrix, over a $50 \mathrm{~mm}$ diameter cylindrical mandrel on a lathe machine tool. The process was carried out by continuous carbon fiber roving passed through an epoxy resin bath and additives, so that the fiber is impregnated with resin. Hoop winding pattern was followed with an approach angle of 90 degrees. Low viscosity epoxy resin was applied to the filament before being wounded and excess resin was wiped out. Figure 1 shows the CFRP tube and the Titanium nitride (TiN) coated, Tungsten Carbide-Cobalt (WC-CO) indexable tool insert used in this work. The CFRP tube was orthogonally turned under dry machining conditions to study the cutting process without any external hindrance. The experimental plan included machining the tube over five different cutting speeds and three different feed rates with a constant depth of cut, in order to study the process under low to high cutting speed and feed rates. The chip microstructure was analyzed using a powerful, high resolution scanning electron microscope (SEM). Table 1 shows the experimental set up and cutting conditions. Fig. 2 shows the experimental layout. The experiments were performed on the Unitech precision lathe

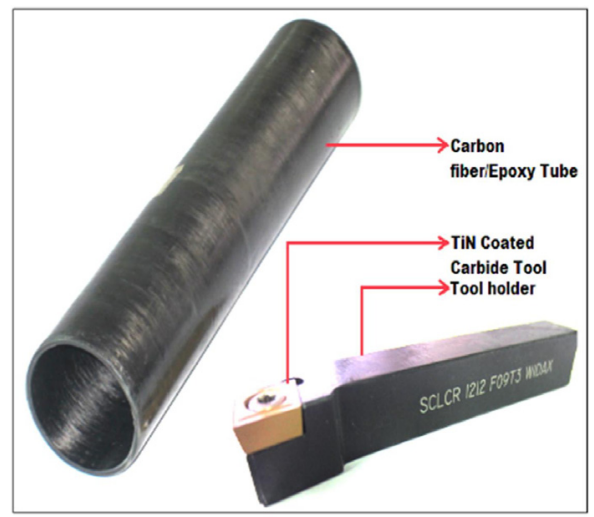

Fig. 1. CFRP tube and tool insert tool. The cutting forces were sensed using a standard calibrated IEICOS strain gauge dynamometer (621C model) with an accuracy of $95 \%$ and data acquisition done with multi component digital force indicator software.

The CFRP tube was fixed in the lathe chuck and other end located using a revolving pipe centre held in the lathe tailstock. The composite chips were collected and analyzed under a high resolution SEM. The chip was mounted on the disk using a carbon tape adhesive and an ion sputter coated with gold alloy was done to get good chip conductivity. The SEM was done at a resolution of $10 \mathrm{~nm}$ and magnification up to $100,000 \times$.

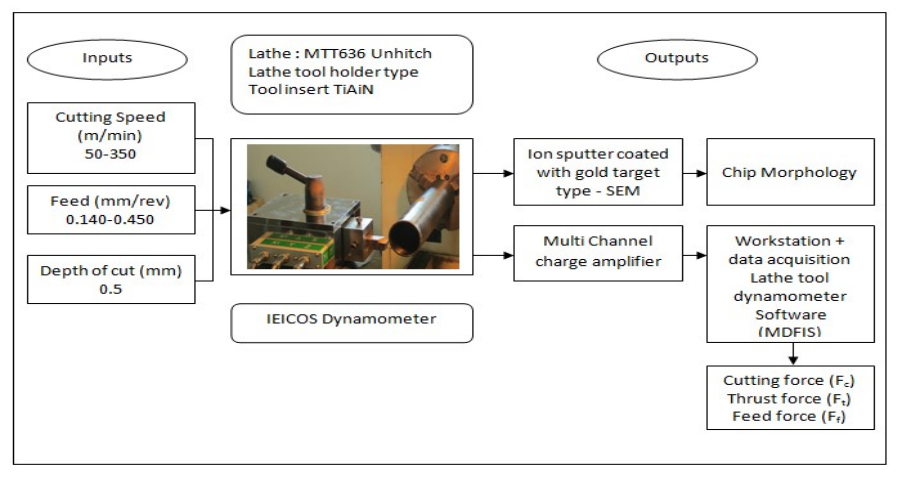

Fig. 2. Experimental Layout

TABLE 1

Experimental set up and cutting conditions

\begin{tabular}{|c|c|}
\hline Factors & Specifications \\
\hline $\begin{array}{c}\text { Work Dimension }(\mathrm{OD} \times \mathrm{ID} \times \mathrm{L}) \\
\text { mm }\end{array}$ & $55 \times 50 \times 260$ \\
\hline Fiber orientation $\left({ }^{\circ}\right)$ & 90 \\
\hline Cutting Speeds $(\mathrm{m} / \mathrm{min})$ & $52,79,130,217,346$ \\
\hline Feed Rate $(\mathrm{mm} / \mathrm{rev})$ & $0.149,0.243,0.446$ \\
\hline Depth of Cut $(\mathrm{mm})$ & 0.5 \\
\hline Environment & Dry condition \\
\hline Tool Holder & WIDAX SCLCR 1212 F09T3 \\
\hline Insert & CCMT 09T304 \\
\hline Tool Material & TiN Coated WC - CO, Tool insert \\
\hline Rake Angle $\left(^{\circ}\right)$ & 7 \\
\hline Clearance Angle $\left(^{\circ}\right)$ & IEICOS \\
\hline Dynamometer & MTT 636 - Unitech Lathe \\
\hline Lathe &
\end{tabular}

\section{Results and discussions}

\subsection{Cutting Forces Vs Cutting Speed}

Cutting forces in machining CFRP materials show marked differences compared to conventional materials. The cutting forces generally decrease with increasing cutting speeds $\left(V_{c}\right)$ irrespective of the feed rates $(f)$ or depth of cuts in conventional material machining, however in CFRP machining, the forces show significant variations with respect to cutting speeds at different feed rates due to the anisotropic nature of the composite material. The CFRP material showed contrasting cutting force 
characteristics at low, medium and high cutting speeds. The material shows brittle fracture behaviour at low cutting speeds affecting its machinability, however increasing cutting speeds leads to two opposing effects on the process: it raises the strain rate, resulting in low strain, brittle fracture, while it raises the temperature in the cutting zone, thereby enhancing the long range mobility of the material's molecular chains, causing ductility. This change in material deformation behaviour at low to high cutting speeds, leads to a highly fluctuating cutting force pattern.

The principal cutting forces $(\mathrm{Fz})$ affect the power and specific cutting energy of the machining process, but the thrust forces $(F y)$ which reflect the interaction of the machined surface and the tool clearance angle and the feed forces $(F x)$ which indicate the effect of tool feed (perpendicular to both the cutting and thrust forces) affect the deformation characteristics of the composite material as well as its machinability.

Figures 3-5, show the plot of cutting forces per unit width (cutting, thrust and feed forces) versus cutting speeds $(V c)$ at different feed rates $(f=0.149 \mathrm{~mm} / \mathrm{rev}, 0.243 \mathrm{~mm} / \mathrm{rev}$, $0.446 \mathrm{~mm} / \mathrm{rev}$ ) respectively. At a low feed rate (Fig. 3) the principal cutting force showed a marked increase with cutting speeds, which is contrary to those observed in conventional machining. The thrust forces, showed higher oscillations at varying cutting speeds, but decreased from a higher value to a lower value with increasing cutting speed. The feed forces also showed some fluctuations, but its mean value was more consistent than the other two forces. The feed forces partially mirrored the effects of the principal cutting forces. The principal cutting force at a medium feed rate of $0.243 \mathrm{~mm} / \mathrm{rev}$ (Fig. 4) decreased with cutting speeds similar to conventional materials showing no fluctuations, while the thrust and feed forces oscillated at a medium cutting speed and thereafter decreased with increasing cutting speeds similar to the cutting forces. With increasing feed rate of $0.446 \mathrm{~mm} / \mathrm{rev}$ (Fig. 5), the principal cutting force showed a gradually descending trend with cutting speeds with minimal oscillations, while the thrust forces and feed forces showed significant fluctuations at low and medium cutting speeds respectively. The higher feed rates have a greater influence on the thrust and feed forces, while principal cutting forces remain unaffected. Waviness is caused by instabilities in cutting process. It depends on the parameter of cutting speed; it may appear randomly because of irregularities in cutting speed. It was observed that while machining, increase in cutting speed generally results in an increase in surface waviness.

The CFRP material probably behaves more like a conventional material at high feed rates in replicating the principal cutting force characteristics suggesting a tendency to become more ductile due to higher temperatures and better molecular mobility. However higher fluctuations at lower feed rates could be a minor hiccup. But higher oscillations of feed and thrust forces pose a problem which could affect the dimensional accuracy and machinability of the composite material. The low thermal conductivity of the material adds to the woes of machining this material, since lack of quick heat transfer from the machined surface and composite chip accelerates tool wear.

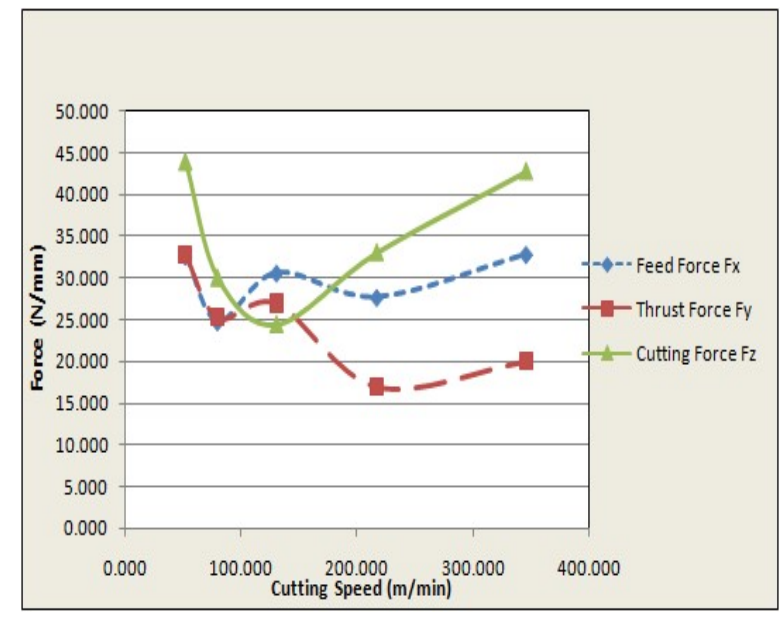

Fig. 3. Cutting forces, $f=0.149 \mathrm{~mm} / \mathrm{rev}$

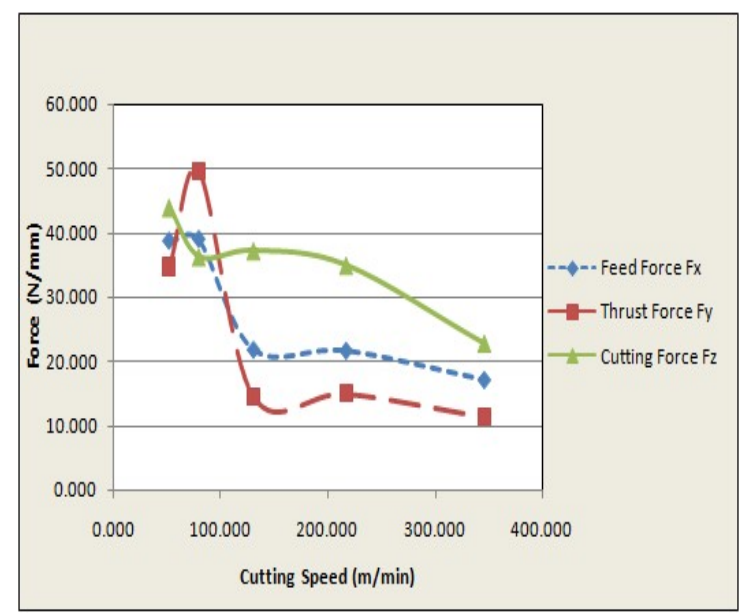

Fig. 4. Cutting forces, $f=0.243 \mathrm{~mm} / \mathrm{rev}$

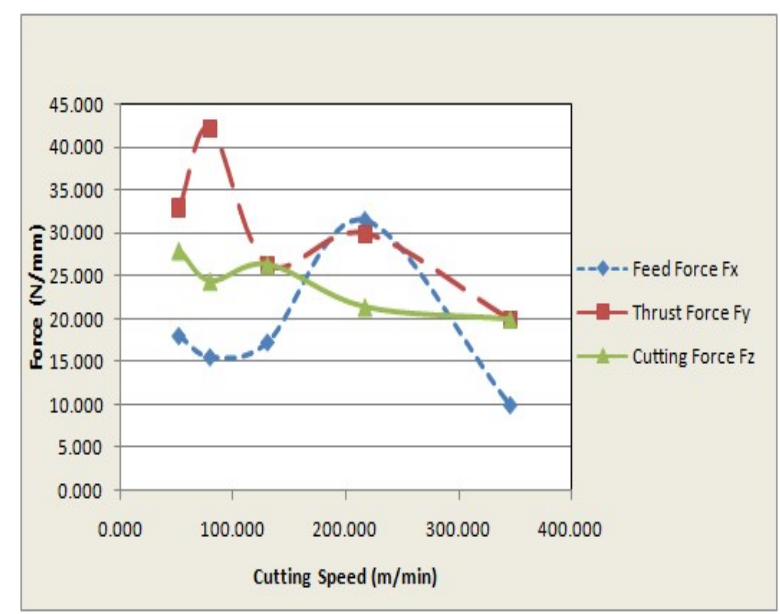

Fig. 5. Cutting forces, $f=0.446 \mathrm{~mm} / \mathrm{rev}$

\subsection{Cutting Forces vs Feed rate}

The cutting forces in CFRP machining are also impacted by feed rates, unlike in conventional machining processess where cutting speeds are more important. The feed rates and the depth of cut induce vibrations in the machine tool which affects the 
stability and dimensional accuracy of the machining process. Unlike metals, composites induce more vibrations during the cutting process even at low feed rates due to material inhomogenity.

Figures 6-10 show the plots of cutting forces vs feed rates over different cutting speeds. At a cutting speed of $52 \mathrm{~m} / \mathrm{min}$, the principal cutting force $(F z)$ and feed force $(F x)$ generally decrease with feed rates, while the thrust forces $(F y)$ appear fairly constant across feed rates. However an increase in thrust force is observed at a slightly elevated cutting speed of $79 \mathrm{~m} / \mathrm{min}$ (Fig. 7), while the principal cutting force and feed force show a trend similar to the trend observed at a cutting speed of $52 \mathrm{~m} / \mathrm{min}$. The principal cutting force magnitude is higher at a cuting speed of $130 \mathrm{~m} / \mathrm{min}$ (Fig. 8) but the variations with feed rate indicate an enhanced principal cutting force at a medium feed rate of $0.243 \mathrm{~mm} / \mathrm{rev}$. The feed and thrust forces show a decrease at $0.243 \mathrm{~mm} / \mathrm{rev}$, gradually increasing at higher feed rates.The trend seen at a cutting speed of $130 \mathrm{~m} / \mathrm{min}$ (Fig. 9) is common with the observation at $217 \mathrm{~m} / \mathrm{min}$, with the feed and thrust forces showing same magnitude at the highest feed rate of $0.446 \mathrm{~mm} / \mathrm{rev}$. At the highest cutting speed of $346 \mathrm{~m} / \mathrm{min}$ (Fig. 10), the cutting forces gradually decrease with feed rates similar to the trend observed

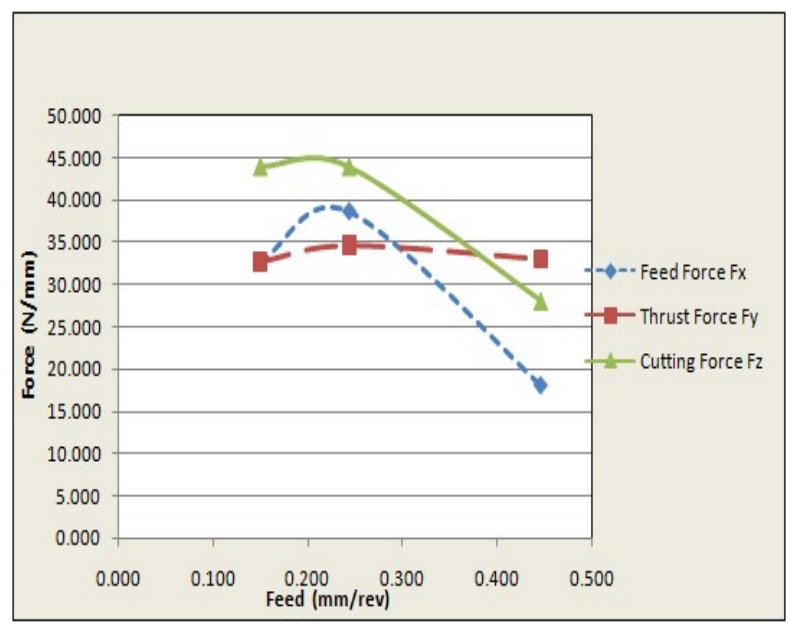

Fig. 6. Cutting forces, $V_{c}=52 \mathrm{~m} / \mathrm{min}$

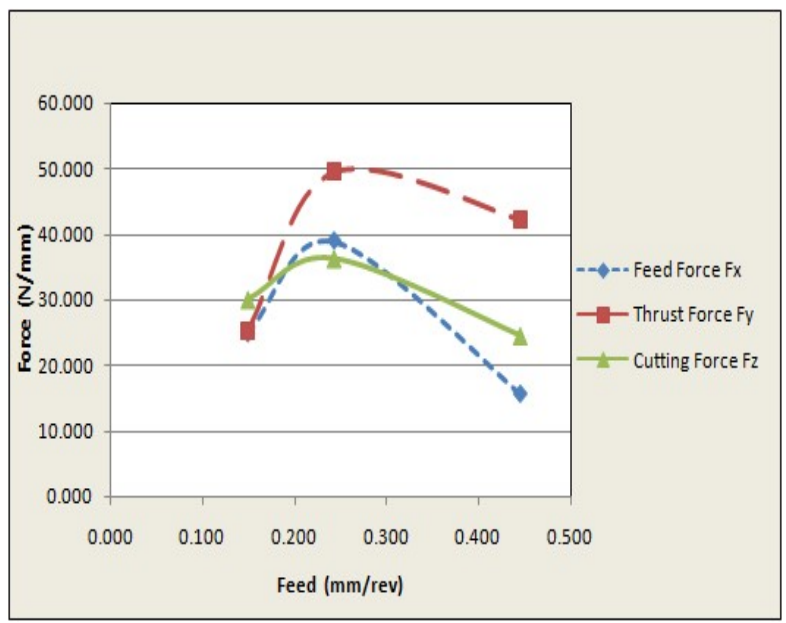

Fig. 7. Cutting forces, $V_{c}=79 \mathrm{~m} / \mathrm{min}$

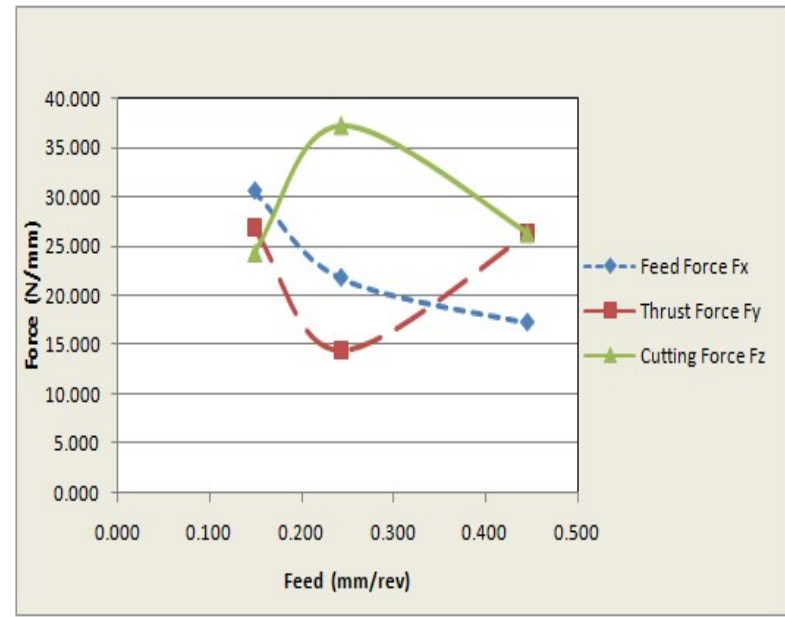

Fig. 8. Cutting forces, $V c=130 \mathrm{~m} / \mathrm{min}$

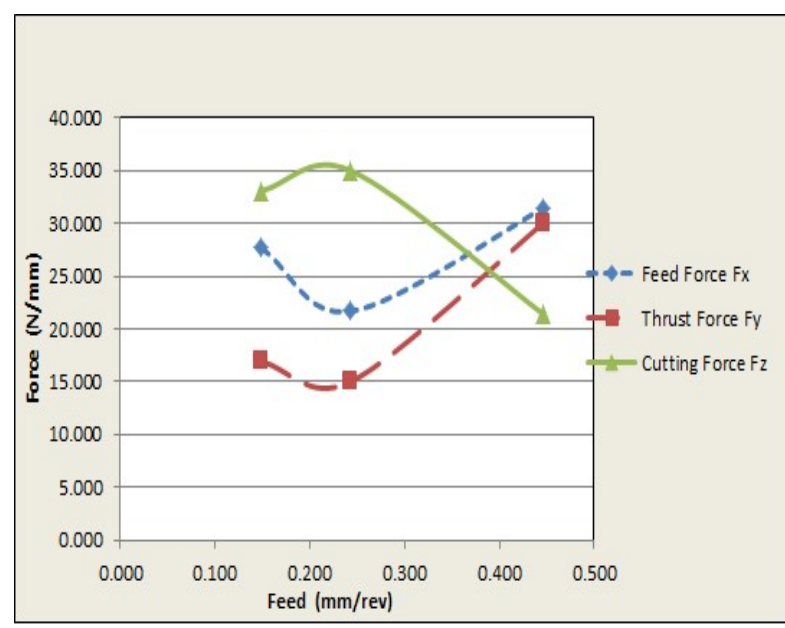

Fig. 9. Cutting forces, $V c=217 \mathrm{~m} / \mathrm{min}$

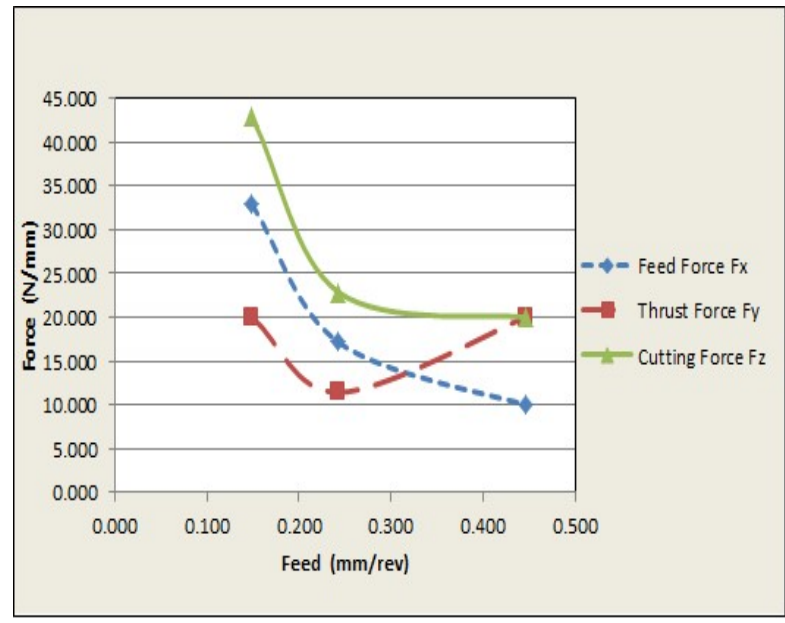

Fig. 10. Cutting forces, $V c=346 \mathrm{~m} / \mathrm{min}$

with cutting speeds, with the feed forces replicating the principal cutting force trend.However a marginal increase in thrust forces were observed at a feed rate of $0.446 \mathrm{~mm} / \mathrm{rev}$.

The observation shows that all the three forces are impacted by feed rates, but the oscillations are not as high as with respect to cutting speeds. The cutting forces appear to follow a descending 
curve as feed rates increase showing the dominance of ductile transition in the composite polymer, making machinability easier. However thrust forces are inconsistent with feed rates and dominate principal cutting force at intermediate cutting speeds. The decrease of the principal cutting forces with increasing feed rates, across cutting speeds are indicative of the need to machine these materials at enhanced feed rates.

\subsection{Chip microstructure}

Chip formation in metals and polymers are vital sources of information on the deformation characteristics of the materials in a machining process, more so with composite materials where the fiber and matrix have unique structural features and distinct fracture and failure criterions. Very little study has been done on chip microstructure changes with respect to cutting parameters in machining. The chips formed at various cutting conditions were collected and observed under an SEM to reveal the micro structural changes of the fiber and matrix layers at various stages of the cutting process. The CFRP material is more prone to debonding due to the self lubricating property of the carbon fiber which helps the fiber to glide over the epoxy matrix. At high cutting speeds and low feed rates, the epoxy matrix has a tendency to fracture early due to high strain rates resulting in smaller chip segments.

In machining of CFRP, the mechanism associated with the material was shearing and rupturing. In the orientation towards 90 degrees, the inter laminar shear increases, leading to fracture of the chip segments along the fiber-matrix interface. Fig. 11 shows the chip microstructure at the highest cutting speed of $346 \mathrm{~m} / \mathrm{min}$ and highest feed rate of $0.446 \mathrm{~mm} / \mathrm{rev}$. The instantaneous fracture of the long continuous strands of carbon fiber is prominent showing significant fiber debonding at intermittent distances on the chip. The higher cutting speeds and feed rates causes quick and instantaneous fracture of the fiber and matrix layers which leads to improved machinability, lower cutting forces, lower specific cutting energy and power required. At a marginally lower feed rate (Fig. 12), at the same cutting speed, significant epoxy matrix debonding is observed with fiber pull out. In high cutting speed and feed it has been observed that, due to high temperature at work - tool interface the adhesion strength between fiber and matrix can be exceeded, so that fibers either peel (delamination) or are removed by the expansion of matrix parts. As the cutting speed decreases to $217 \mathrm{~m} / \mathrm{min}$, (Fig. 13) the fibers break in small fractions showing higher deformation towards the outer surface than the inner core suggesting that marginally higher cutting forces are required to cause fracture. Also fiber delamination and pull out is a prominent feature at this intermediate cutting speed with the fibers showing out of plane cracking compared to the failure at higher cutting speeds where the fiber fracture was associated with in plane cracking. Fig. 14, shows an interesting phenomena of chip segmentation which is normally seen in high speed machining of metals, more so with titanium alloys (even at low speeds) which are also

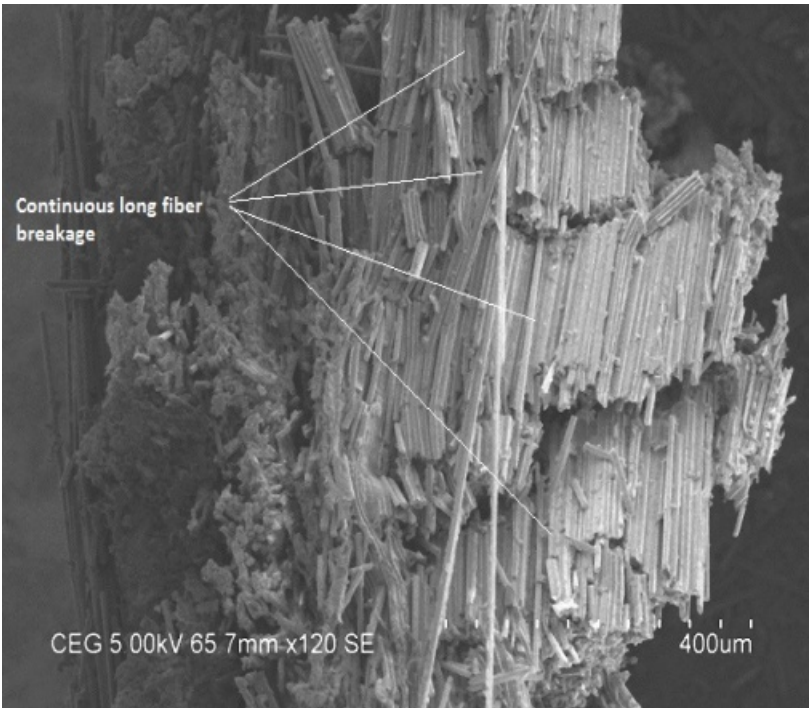

Fig. 11. Chip micrograph $-V c=346 \mathrm{~m} / \mathrm{min}, f=0.446 \mathrm{~mm} / \mathrm{rev}$

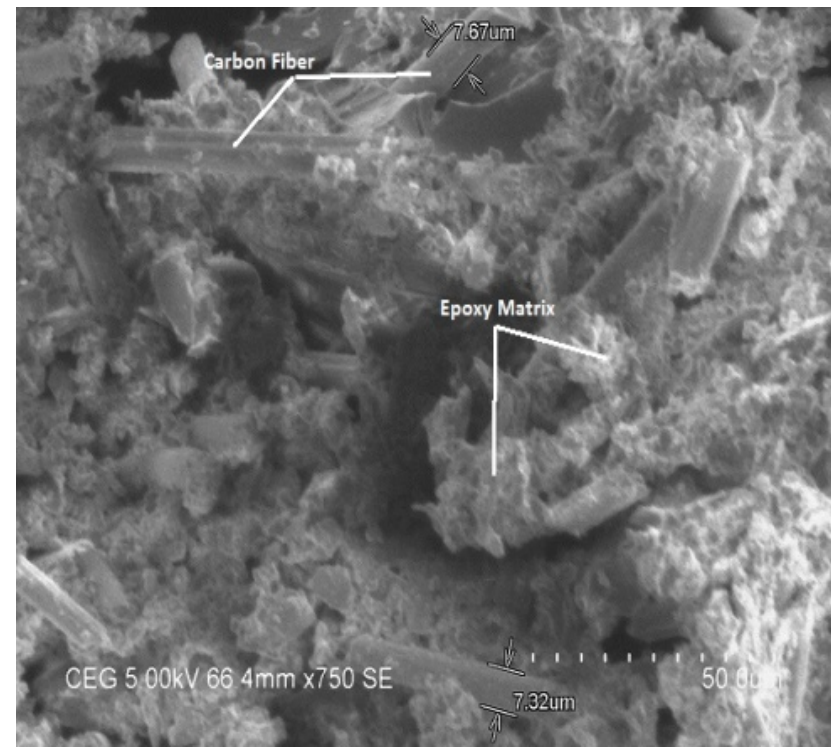

Fig. 12. Chip micrograph $-V c=346 \mathrm{~m} / \mathrm{min}, f=0.243 \mathrm{~mm} / \mathrm{rev}$

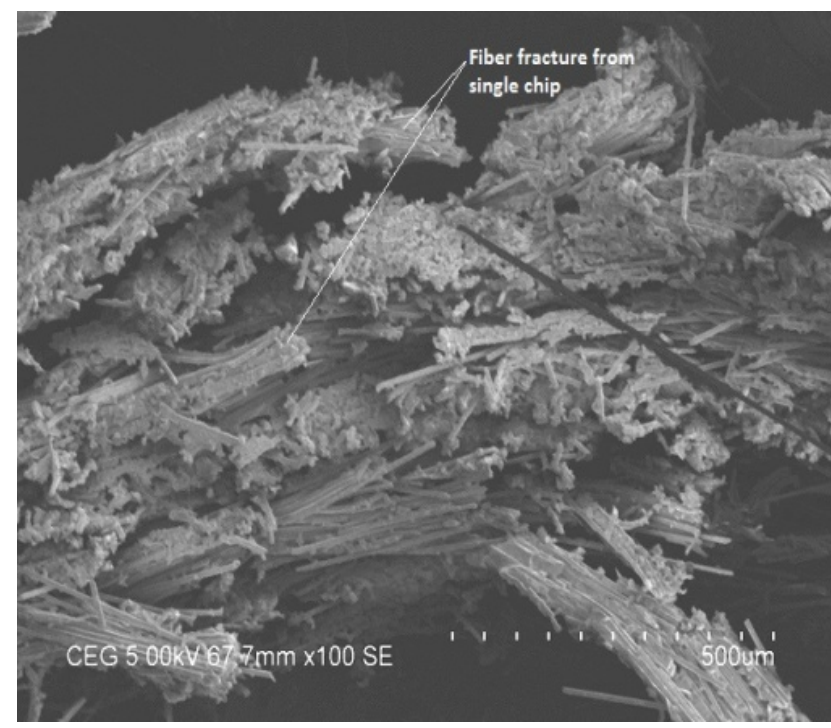

Fig. 13. Chip micrograph $-V_{c}=217 \mathrm{~m} / \mathrm{min}, f=0.243 \mathrm{~mm} / \mathrm{rev}$ 
classified as difficult to machine materials like composites. The chip micrograph at this cutting speed $(130 \mathrm{~m} / \mathrm{min})$ and highest feed rate $(0.446 \mathrm{~mm} / \mathrm{rev})$ show serrations and segmentations at the periphery of the chip with higher surface damage than the

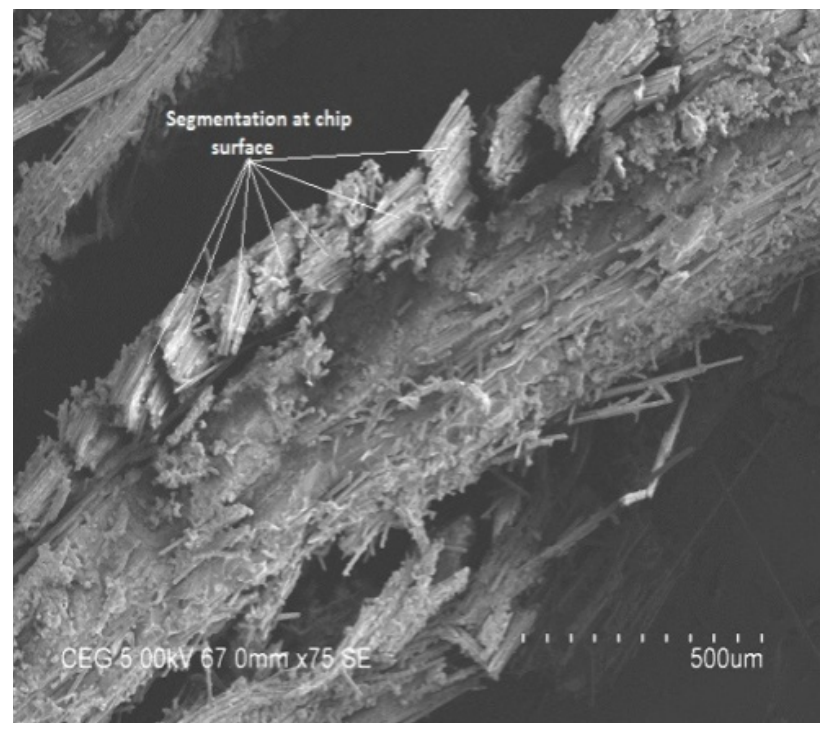

Fig. 14. Chip micrograph $-V_{C}=130 \mathrm{~m} / \mathrm{min}, f=0.446 \mathrm{~mm} / \mathrm{rev}$

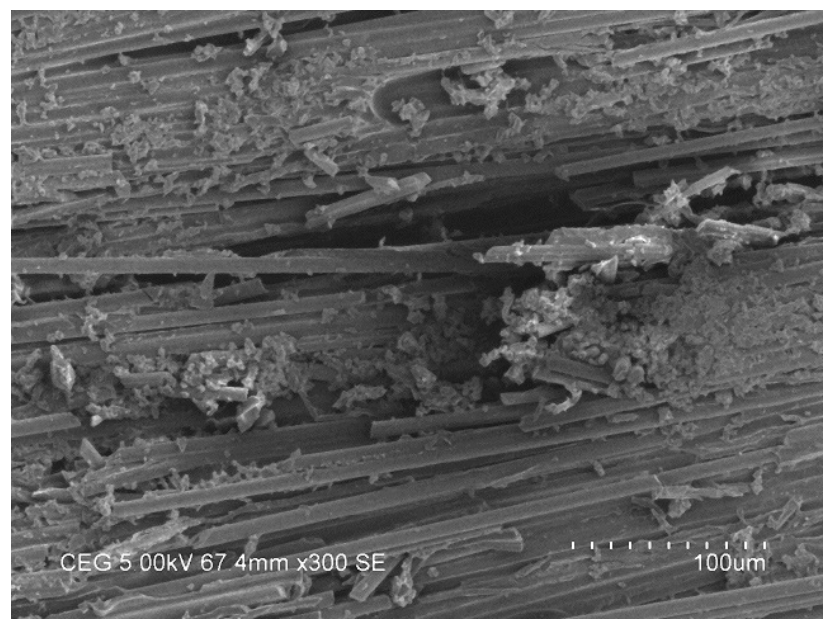

Fig. 15. Chip micrograph $-V c=79 \mathrm{~m} / \mathrm{min}, f=0.149 \mathrm{~mm} / \mathrm{rev}$

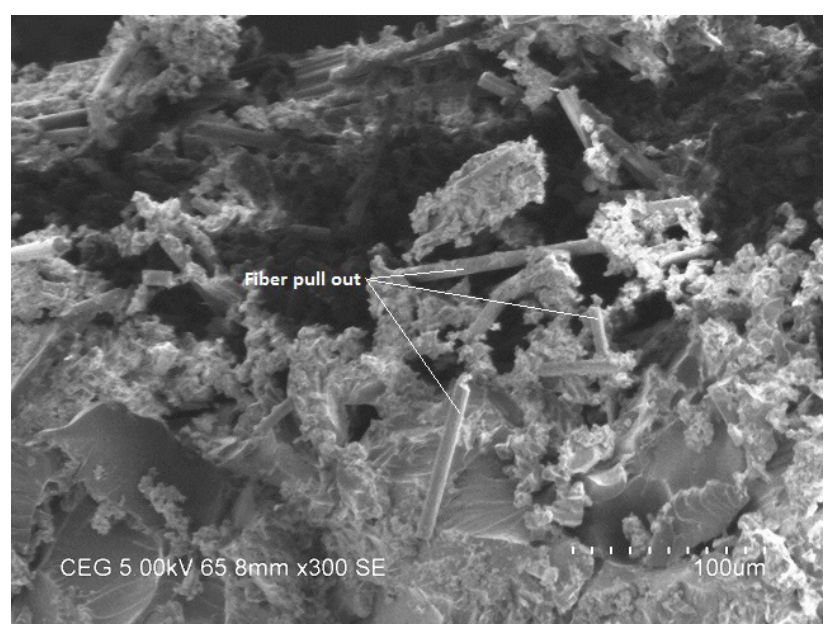

Fig. 16. Chip micrograph $-V c=52 \mathrm{~m} / \mathrm{min}, f=0.446 \mathrm{~mm} / \mathrm{rev}$ inner core with individual facets inclined to the fiber axis. The combination of low speed and high feed rate leads to higher oscillations during the cutting process which could lead to the chip segmentation.

At even lower cutting speed of $79 \mathrm{~m} / \mathrm{min}$ and feed rate of $0.149 \mathrm{~mm} / \mathrm{rev}$ (Fig. 15), the segmentation pattern is absent and the fibers show only marginal failure causing higher force requirements and poor machinability. Even the matrix phase shows very little sub surface damage leading to lower fiber fracture as well. However when the cutting speeds are the lowest at $52 \mathrm{~m} / \mathrm{min}$ and highest feed rate of $0.446 \mathrm{~mm} / \mathrm{rev}$, significant failure of the matrix is observed (Fig. 16) with fiber pull out as well. The higher feed rates in this case must have resulted in the matrix damage and fiber pull out. This indicates that during machining, when the CFRP composite is stressed, might have caused easy gliding of the fibre through the matrix, i.e., the carbon-matrix bond may not be as strong. This may also be a factor for getting the good surface texture of machined CFRP surfaces.

The chip microstructure across cutting speeds and feed rates suggest that different modes of failure of the carbon fiber and epoxy matrix are observed at different machining conditions and no two conditions produce similar chip patterns. However, higher cutting speeds and feed rates cause instantaneous fiber fracture with associated matrix failure which helps in the machining process.

\section{Conclusions}

The objective of this research work was to analyze the impact of cutting conditions on the cutting forces and chip microstructure during machining of a CFRP material in tube form. The feed rates have a prominent impact on the three forces, and cause significant fluctuations in thrust and feed forces especially at low feed rates. The thrust forces dominate the principal cutting force and feed force at medium and higher feed rates. Cutting speeds impact the principal cutting force less at higher feed rates, but show high oscillations of feed and thrust forces, with thrust forces continually increasing with feed rates at higher cutting speeds. The cutting speed and feed rates affect the thrust forces across different conditions and have a significant bearing on the machinability of the composite material. The principal cutting force trend is similar to conventional material machining especially at higher cutting speeds. Feed force impact is lesser than thrust forces.

The microscopic examination of the chips showed the impact of cutting conditions on the chip characteristics. Instantaneous brittle fracture of the carbon fiber was observed at high cutting speeds and feed rates with little fiber pullout. Significant epoxy matrix damage was observed at marginally lower feed rates and high cutting speeds. Interestingly chip segmentation across the periphery of the chip was observed at low cutting speed and higher feed rates. Fiber pullout and matrix damage, with fractured fibers firmly embedded in the matrix was observed at the lowest cutting speed. The CFRP 
material shows a duality in material behaviour with brittleness as well as ductility characteristics at different cutting conditions. This coupled with their anisotropic property and low thermal conductivity makes these materials poor choices for machining processes. However, high speed machining can be employed at optimized feed rates and lower depths of cut to successfully machine these materials.

\section{Acknowledgement}

The authors wish to express their gratitude to AICTE, Govt. of India for sponsoring this research project under a Research Promotion Scheme (RPS) grant.

\section{REFERENCES}

[1] R. Ch. Dandekar, C.Y. Shin, Int. J. Mach. Tool 57, 102-121 (2012).

[2] Ch.-S. Chang, J. Mater. Process. Tech. 180, 117-129 ( 2006).

[3] C. Dold, M. Henerichs, L. Bochmann, K. Wegener, Procedia CIRP, 178-183 (2012).
[4] K.S. Kim, D.G. Lee, K. Kwak, S. Namgung, J. Mater. Process. Tech. 32, 553-570 (1992).

[5] O. Pecat, R. Rentsch, E. Brinksmeier, Procedia CIRP, 466-470 (2012).

[6] R. Rusinek, Int. J. Non Linear Mech. 45, 458-462 (2010).

[7] M. Rahman, S Ramakrishna, J.R.S. Prakash, D.C.G. Tan, J. Mater. Process. Tech. 89-90, 292-297 (1999).

[8] Rajasekaran, Palanikumar, Vinayagam Procedia Eng. 38, 29222929 (2012).

[9] R. Rentsch, O. Pecat, E. Brinksmeier, Procedia Eng. 10, 1823-1828 (2011).

[10] G. Santhanakrishnan, R. Krishnamurthy, S.K. Malhotra, Investigation into the machining of carbon- fiber reinforced plastics with cemented carbides, J. Mater. Process. Tech. 300, 263-275 (1992).

[11] T. Yashiro, T. Ogawa, H. Sasahara, Int. J. Mach. Tool Manu. 70, 63-69 (2013).

[12] R. Teti, CIRP Annals-Manu. Tech. 51, 611-634 (2012).

[13] V. Krishnaraj, Prabukarthi, A. Ramanathan, Elanghovan, S. Kumar, R. Zitoune, Davim, Composites: Part B , pp.1791-1799 (2012).

[14] X.M. Wang, L.C. Zhang, Int. J. Mach. Tool Manu. 43, 1015-1022 (2003).

[15] Y. Karpat. N. Polat, CIRP Ann-Manu. Tech. 62, 95-98 (2013). 